\title{
Las tecleras o clickers como un instrumento complementario que favorece la construcción de Aprendizaje Significativo en la resolución de problemas en electromagnetismo
}

Rafael Silva Córdova ${ }^{1}$

Departamento de Física - Universidad de Playa Ancha

Ester López Donoso ${ }^{1}$

Centro de Estudio Avanzados - Universidad de Playa Ancha

Valparaíso - Chile

\section{Resumen}

Una de las dificultades que tiene la teoría del aprendizaje significativo, es poder determinar en forma inmediata la asimilación conceptual, es decir, saber cómo el aprendiz está interactuando entre los conceptos previos y los conceptos nuevos por aprender. Las tecleras o clickers como instrumento tecnológico de las nuevas tecnologías de la información y comunicación, permiten poder tener respuestas en forma instantánea de cada uno de los estudiantes cuando son sometidos a preguntas que expliciten su proceso de asimilación. Esta investigación propone un método de enseñanza en la resolución de problema, basada en la indagación guiada, de manera que el profesor detecte la asimilación de los estudiantes cuando estos resuelven problemas. Se sustenta en la teoría del aprendizaje significativo de Ausubel y en la teoría sociocultural de Vygotsky. La investigación se resuelve mediante una metodología mixta, cuantitativa y cualitativa, con un diseño cuasiexperimental, donde se comparan dos grupos en la resolución de problemas; un grupo control y otro experimental. La resolución de problema se realiza en un curso de Electromagnetismo, a nivel de carreras de pregrado universitario. Los resultados muestran avance importante en la adquisición de aprendizaje significativo entre el grupo experimental y grupo control.

\footnotetext{
${ }^{+}$Keyboards or clickers as a complementary instrument that favors the construction of Meaningful Learning in the resolution of problems in electromagnetism

* Recebido: julho de 2019. Aceito: julho de 2020.

${ }^{1}$ E-mails: rsilva@upla.cl; elopez@upla.cl
} 
Palabras clave: Aprendizaje Significativo; Asimilación; Uso de Tecleras; Resolución de Problemas; Indagación Guiada.

\begin{abstract}
One of the difficulties that the theory of significant learning has is to be able to immediately determine conceptual assimilation, that is, to know how the learner is interacting between the previous concepts and the new concepts to be learned. The tecleras or clickers as a technological instrument of the new information and communication technologies allow us to have instantaneous answers from each one of the students when they are subjected to questions that explain their assimilation process. This research proposes a teaching method in problem solving, based on guided inquiry, so that the teacher detects the assimilation of the students when they solve problems. It is underpinned by Ausubel's theory of meaningful learning and Vygotsky's sociocultural theory. The investigation is solved by means of a mixed, quantitative and qualitative methodology, with a quasi-experimental design, where two groups are compared in problem solving; a control group and an experimental group. Problem solving is carried out in an Electromagnetism course, at university undergraduate level. The results show significant progress in the acquisition of significant learning between the experimental group and the control group.
\end{abstract}

Keywords: Meaningful Learning; Assimilation; Tecleras Use; Problem Solving; Guided Inquiry.

\title{
I. Introducción
}

El uso de las Tecnologías de la Información y la Comunicación (TIC) se ha generalizado en todos los ámbitos de la vida, entre ellos, la formación de profesionales. Cada vez es mayor el número de instituciones de educación superior que utilizan este tipo de herramientas con fines de enseñanza. La oferta educativa apoyada en TIC (ya sea virtual o bimodal) ha ido creciendo; sin embargo, no siempre encontramos propuestas educativas planeadas, y bien fundamentadas, dado que proliferan alternativas que replican un modelo tradicional de enseñanza y promueven un aprendizaje memorístico y descontextualizado, con un diseño tecno pedagógico poco innovador. Por ello, la presente investigación se propone utilizar las tecleras como un medio para provocar la asimilación y anclaje adecuado, que permita la adquisición de aprendizaje significativo en electromagnetismo, a nivel de pregrado. 
Para que haya aprendizaje significativo, según la Teoría del Aprendizaje Significativo de Ausubel (MOREIRA, 2011), el material que el alumno tiene que aprender debe ser potencialmente significativo, es decir, relacione en su estructura cognitiva de forma no arbitraria y no literal, y manifieste una predisposición para aprender, o sea, una disposición a relacionar el nuevo material de manera sustantiva y no arbitraria en su estructura de cognitiva (MOREIRA, 2011; MOREIRA; MASINI, 2006).

La Teoría de los Campos Conceptuales de Vergnaud (1990) es totalmente compatible con la Teoría del Aprendizaje Significativo de Ausubel. Para Vergnaud, la esencia del desarrollo cognitivo es la conceptualización (MOREIRA, 2004). La conceptualización es un proceso a través del cual los conceptos se hacen significativos a través de una variedad de situaciones, las cuales dan sentido a los conceptos. Por tanto, es natural definir campo conceptual, sobre todo, como un conjunto de situaciones (op. cit.). En esa teoría, la importancia de la acción del sujeto es enfatizada como mediadora de la evolución conceptual, por tanto, es importante ofrecerles a los alumnos una gran cantidad de situaciones con diferentes grados de complejidad (CABALLERO; MOREIRA; RODRÍGUEZ, 2008). Por ello, es fundamental la elección de los problemas que los alumnos deben resolver.

La presente investigación se propone utilizar las tecleras con un medio para producir una asimilación y anclaje adecuado, que permita la adquisición de aprendizaje significativo en electromagnetismo. La investigación es un diseño cuasi experimento, donde se comparan dos grupos de enseñanza en la resolución de problemas de Electromagnetismo, en cursos de pregrado. El curso control (con 24 alumnos) sigue una metodología de enseñanza bajo una pedagogía activa, sustentada en el aprendizaje significativos con una serie de actividades, basada en el aprendizaje significativo de Ausubel, modelo diseñado por Silva (2011). Las actividades del modelo son: material potencialmente significativo, aplicaciones, simulaciones, webquest, ayudantías, foros de discusión, mapas conceptuales y talleres de resolución de problemas. El curso experimental (con 48 alumnos) tiene el mismo modelo de enseñanza pero se diferencia del anterior en el taller de resolución de problemas. En este taller de resolución de problemas, se utiliza una metodología de enseñanza indagatoria guiada, donde cada grupo debe resolver dos problemas, que se realizan durante una sesión de 90 minutos, y que deben entregar al final de la sesión. En las otras dos sesiones de clases del curso se desarrollan el resto de las actividades. El curso de Electromagnetismo tiene tres unidades temáticas: Electrostática, circuitos eléctrico, y magnetismo. Se han realizado 4 talleres de resolución de problemas por cada unidad temática.

La metodología indagatoria guiada consiste en ir resolviendo un problema, en grupos de tres alumnos, paso a paso, con un tiempo asignado para ello, y que son evaluados en forma instantánea mediante las tecleras, entregando resultados y análisis en forma inmediata. Esto permite saber qué grupo ha asimilado de buena manera y que grupo ha cometido errores. Por lo tanto, durante un cierto tiempo se hace una discusión de los resultados, negociando significado con todos los integrantes del curso, bajo la conducción del profesor. 
La presente investigación se hace las siguientes preguntas:

¿Se obtendrá mejores rendimientos académico y calidad de aprendizaje cuando se utiliza una metodología indagatoria guiada y apoyada con uso de tecleras en la resolución de problemas, que la metodología tradicionales?

¿Afectará en las pruebas integrales o globales el utilizar una metodología indagatoria guiada con el uso de tecleras en la resolución de problemas

A continuación, se muestran tres figuras del funcionamiento y formas de utilizar las tecleras.
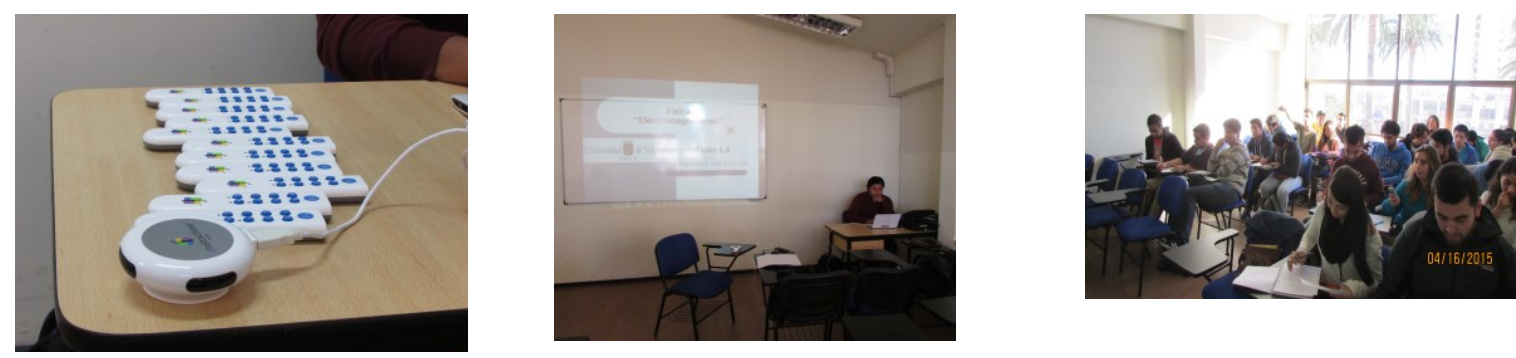

Fig. 1 - Tecleras o clickers utilizadas para la propuesta, en el curso experimental en el desarrollo de un taller.

Según Ausubel, el factor separado más importante que influye en el aprendizaje es lo que ya sabe el aprendiz. Para él, aprendizaje significa organización e integración del nuevo material en la estructura cognitiva. Como otros teóricos del cognitivismo, parte de la premisa de que en la mente del individuo existe una estructura en la cual se procesan la organización y la integración: es la estructura cognitiva, entendida como el contenido total de ideas de un individuo y su organización, o el contenido y organización de sus ideas, en una determinada área de conocimiento. Nuevas ideas e informaciones pueden ser aprendidas y retenidas en la medida en que conceptos, ideas o proposiciones relevantes e inclusivos estén adecuadamente claros y disponibles en la estructura cognitiva del individuo y funcionen, de esa forma, como "ancladero" para nuevas ideas, conceptos o proposiciones. Los organizadores previos pueden tanto suministrar "ideas ancla" relevantes para el aprendizaje significativo del nuevo material, como establecer relaciones entre ideas, proposiciones y conceptos ya existentes en la estructura cognitiva y los contenidos en el material de aprendizaje, o sea, para explicitar la relación que existe entre los nuevos conocimientos y los que el aprendiz ya tiene pero no percibe que se pueden relacionar con los nuevos. Justamente son las tecleras que nos permiten evidenciar si está interacción está ocurriendo, con la participación de todos en la negociación de significados.

Si la condición para que un aprendizaje sea potencialmente significativo es que la nueva información interactúe con la estructura cognitiva previa y que exista una disposición para ello del que aprende, esto implica que el aprendizaje por descubrimiento no 
necesariamente es significativo y que el aprendizaje por recepción sea obligatoriamente mecánico.

Ausubel propone dos principios programáticos de la materia de enseñanza como una consecuencia natural derivada de estos dos procesos de la dinámica de la estructura cognitiva. Es decir, en la búsqueda de organización cognitiva, el ser que aprende va, al mismo tiempo, diferenciando progresivamente y reconciliando entregadoramente los conocimientos adquiridos. Consecuentemente, la enseñanza será más facilitadora del aprendizaje significativo si se consideran estos procesos como principios organizadores. Estos principios son controlados y evaluados permanentemente durante el proceso de esta investigación.

A continuación, se presenta un esquema conceptual, sobre el aprendizaje significativo en el contexto del problema de investigación.

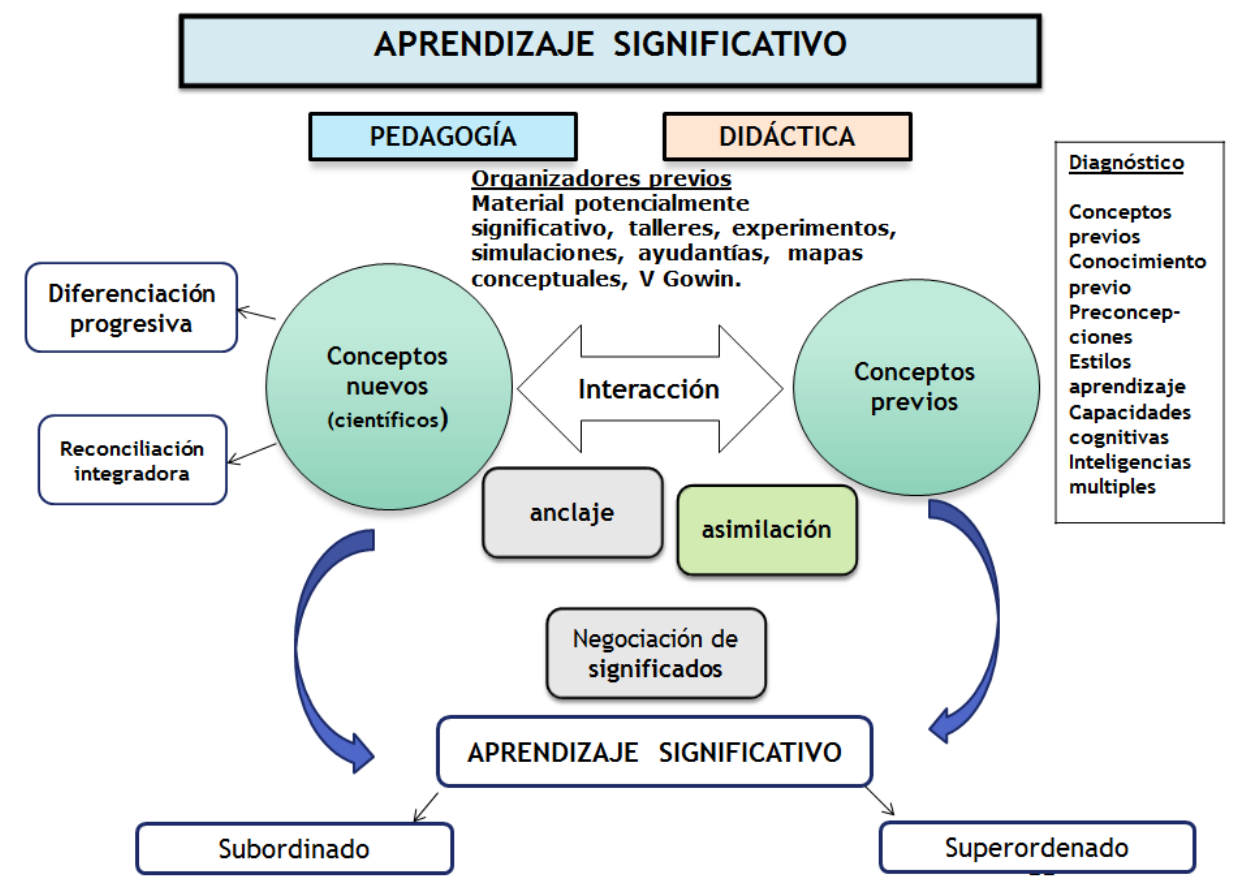

Esquema 1 - Aprendizaje significativo en contexto de uso de tecleras.

Otro referente teórico que complementa al aprendizaje significativo es la Teoría Sociocultural de Vygotsky que sostiene que los estudiantes desarrollan su aprendizaje mediante la interacción social, en la cual ellos van adquiriendo nuevas y mejores habilidades cognitivas. Por ello, es conveniente que el profesor diseñe actividades de talleres de resolución de problemas de manera cooperativa, para que aquellos estudiantes con competencias menores logren habilidades cognitivas con la ayuda de sus compañeros más habilidades, como zona de desarrollo próximo.

En el esquema anterior, se muestra cómo debe operar la metodología de enseñanza. Determinando con precisión los conceptos previos, y las actividades que hagan la interacción 
con los conceptos nuevos. El rol de las tecleras es ir midiendo y evaluando los procesos de asimilación y negociación de significado para producir el anclaje deseado. A través, del lenguaje y la elaboración de mapas conceptuales se podrá verificar la construcción de aprendizaje significativo en sus estudiantes.

A continuación, se presenta un esquema sobre el diseño de la investigación, donde se trata de una metodología cuasi experimental que compara un grupo control y uno experimental en la resolución de problemas de electromagnetismo.

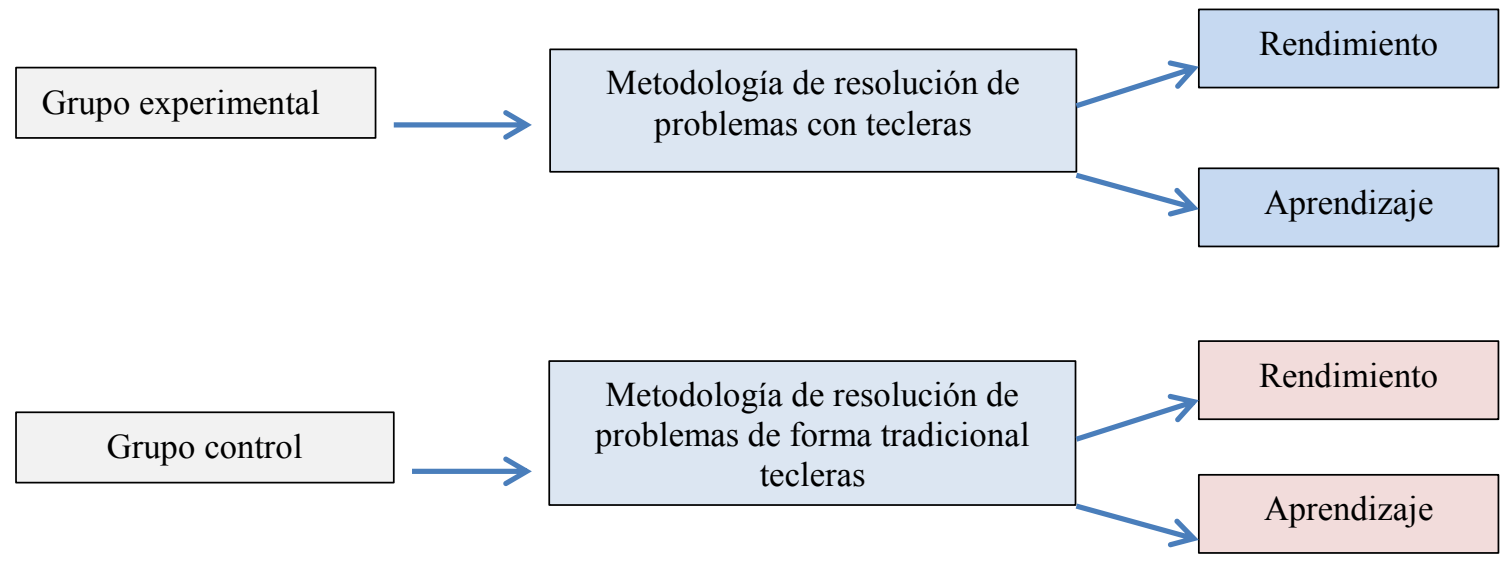

Esquema 2 - Diseño de la investigación.

El rendimiento académico es medido por medio de pautas de corrección, aplicada a ambos grupos experimental y control. El aprendizaje se mide mediante los procedimientos usados en la resolución de problema, determinando que aprendieron y que no aprendieron, complementados mediante la construcción de mapas conceptuales, los registros que van quedando en las tecleras, y el lenguaje usado en la negociación de significados.

\section{Análisis y discusión de resultados}

A continuación, se muestra la metodología indagatoria empleada en la resolución de problema, con aquellos pasos que debe realizar cada grupo, donde cada pregunta es analizada en forma instantánea, produciendo una discusión o negociación de significados en forma inmediata. El problema que muestra como ejemplo, a resolver, corresponde al segundo taller, de la segunda unidad temática, de electricidad, que aborda los circuitos RC. Para mostrar con claridad, la forma de presentar cada problema, pondremos el problema 1 de ese taller, con todas sus preguntas. 
1. Determine la carga máxima acumulada en el condensador y la corriente que circula por $i_{3}$.
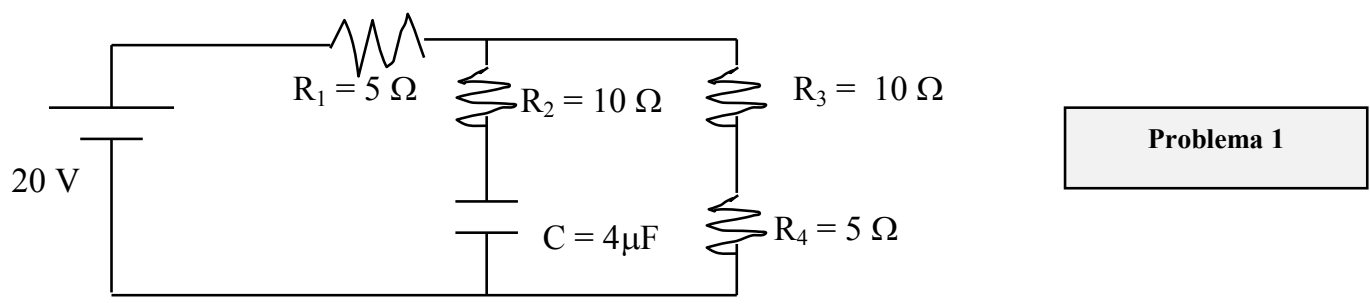

Preguntas:

1.1 ¿Cuál es el valor de $i_{2}$ cuando "C" está cargado completamente?
A) $1[\mathrm{~A}]$
B) $6[\mathrm{~A}]$
C) $0,1[\mathrm{~A}]$
D) $0[\mathrm{~A}]$
E) $0.6[\mathrm{~A}]$

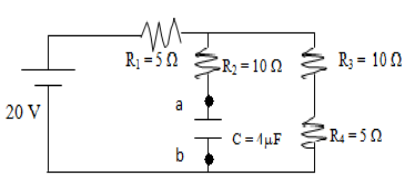

1.2 ¿Cuál es la $\mathrm{R}_{\mathrm{eq}}$ cuando “C” está cargado completamente?
A) $30[\Omega]$
B) $15[\Omega]$
C) $10[\Omega]$
D) $7.5[\Omega]$
E) $20[\Omega]$

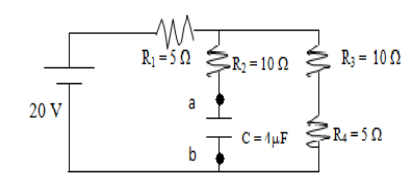

1.3 ¿Cuál es la $i_{\text {eq }}$ cuando "C” está cargado completamente?
A) $10[\mathrm{~A}]$
B) $1[\mathrm{~A}]$
C) $8[\mathrm{~A}]$
D) $5[\mathrm{~A}]$
E) $20[\mathrm{~A}]$

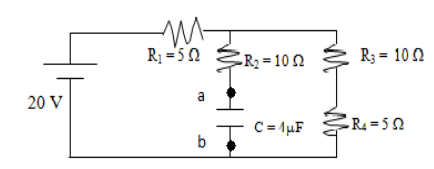

1.4 Determine $\mathrm{Va}-\mathrm{Vb}$.
A) $15[\mathrm{~V}]$
B) $20[\mathrm{~V}]$
C) $25[\mathrm{~V}]$
D) $30[\mathrm{~V}]$
E) $36[\mathrm{~V}]$

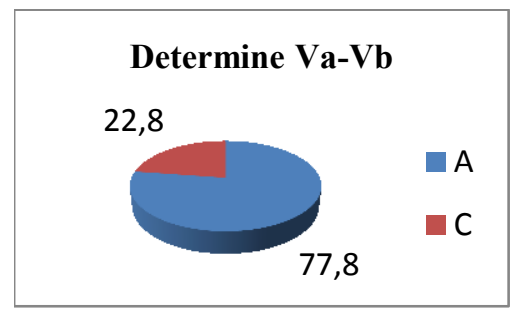


1.5 ¿Cuál es la polaridad en el condensador?
A) Puede ser cualquiera
B) $\frac{\mathrm{a}+}{\mathrm{b}-}$
C) $\frac{\mathrm{a}-}{\mathrm{b}+}$

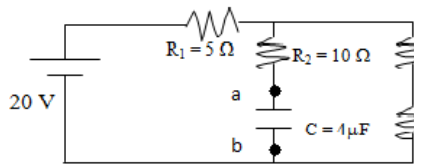
D) $\quad 0$

1.6 ¿Cuál es la qmáx?
A) $60[\mathrm{C}]$
B) $60[\mu \mathrm{C}]$
C) $45[\mathrm{C}]$
D) $45[\mu \mathrm{C}]$
E) $30[\mu \mathrm{C}]$

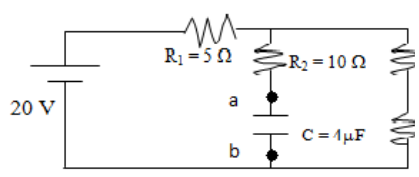

Para poder dar respuesta al estudio comparativo sobre el rendimiento académico entre grupo control y experimental en la resolución de problemas, por cada una de las tres unidades temáticas, del curso de Electromagnetismo. Sus resultados, especialmente en el promedio o media, se muestran a continuación en la siguiente tabla1.

Tabla 1 - Resultados comparativos en resolución de problemas por cada unidad temática.

\begin{tabular}{|cl|c|c|c|c|}
\hline \multicolumn{1}{|c|}{ Grupo } & $\mathrm{N}$ & Media & Desviación típ. & $\%$ Rendimiento \\
\hline \multirow{2}{*}{ Taller $1^{\mathrm{o}}$ unidad } & control & 48 & $\mathbf{5 , 3}$ & 0,40 & $72 \%$ \\
& experimental & 24 & $\mathbf{6 , 1}$ & 0,44 & $85 \%$ \\
\hline \multirow{2}{*}{ Taller 2 ${ }^{\circ}$ unidad } & control & 48 & $\mathbf{5 , 4}$ & 0,36 & $73 \%$ \\
& experimental & 24 & $\mathbf{6 , 3}$ & 0,52 & $88 \%$ \\
\hline \multirow{2}{*}{ Taller 3 ${ }^{\circ}$ unidad } & control & 48 & $\mathbf{5 , 4}$ & 0,33 & $73 \%$ \\
& experimental & 24 & $\mathbf{6 , 5}$ & 0,53 & $92 \%$ \\
\hline
\end{tabular}

Para visualizar de manera gráfica, la comparación del rendimiento entre grupo control y experimental en la resolución de problema se muestra la gráfica 1 , los resultados obtenidos por cada unidad temática. 


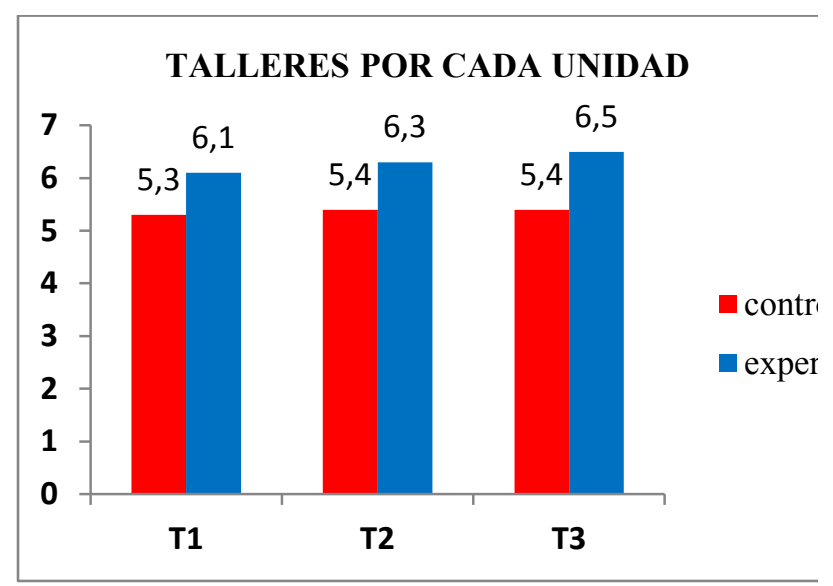

Gráfico 1 - Comparación de resultados de aprendizaje en resolución de problemas por unidad.

Es claro, que la metodología experimental tiene mejores rendimiento académicos que la metodología tradicional, en la resolución de problemas en Electromagnetismo.

Para poder concluir estas diferencias entre los valores obtenidos por cada unidad temática, debido a la intervención en la metodología de enseñanza, es necesario utilizar una estadística paramétrica de comparación, llamada prueba " $t$ ", que debe cumplir ciertas condiciones como la igualdad de varianza y la distribución normal de cada grupo. En caso, que no se cumplan las condiciones anteriores debe usarse una estadística no-paramétrica de comparación, prueba U de Mann Whitney.

La prueba " $t$ ", que muestra diferencia entre grupo control y experimental, se presenta a continuación:

Tabla 2 - Prueba “t” entre grupo control y experimental, por unidad temática.

\begin{tabular}{|l|c|c|c|}
\hline & “t” & $\mathrm{gl}$ & $\begin{array}{c}\text { Diferencias } \\
\text { media }\end{array}$ \\
\hline Taller 1 unidad & $\mathbf{7 , 4 8}$ & 70 & $-0,77$ \\
\hline Taller 2 unidad & $\mathbf{8 , 0 9}$ & 70 & $-0,84$ \\
\hline Taller 3 unidad & $\mathbf{1 0 , 3 4}$ & 70 & $-1,05$ \\
\hline
\end{tabular}

Para 70 grados de libertad la prueba " $t$ ", tiene un valor comprendido en el intervalo $1,67<\mathrm{t}<1,67$. Por lo tanto, queda claro que la propuesta, tiene diferencias relevantes entre un método de resolución de problema y el otro dado por el grupo control.

Como, las condiciones que deben aplicarse a la prueba "t" no se cumplen nítidamente, se confirma con la prueba no paramétrica " $U$ " de Mann-Whitney. Los resultados se muestran en la siguiente tabla. 
Tabla 3 - Estadísticos de contraste(a) prueba “U”, compara grupo control con experimental.

\begin{tabular}{|l|c|c|c|}
\hline & $\begin{array}{c}\text { Taller 1 } \\
\text { unidad }\end{array}$ & $\begin{array}{c}\text { Taller 2 } \\
\text { unidad }\end{array}$ & $\begin{array}{c}\text { Taller 3 } \\
\text { unidad }\end{array}$ \\
\hline U de Mann-Whitney & 99,000 & 117,000 & 69,000 \\
W de Wilcoxon & 1275,000 & 1293,000 & 1245,000 \\
$\mathbf{Z}$ & $\mathbf{- 5 , 7 2}$ & $\mathbf{- 5 , 5 0}$ & $\mathbf{- 6 , 0 8}$ \\
Sig. asintót. (bilateral) &, 000 &, 000 &, 000 \\
\hline
\end{tabular}

Como se trata de muestra grandes, mayores a 20. El valor de $\mathrm{Z}$ debe estar en el intervalo entre $-1,96<Z<1,96$. Como los valores obtenidos para cada unidad están fuera de este rango, confirma efectivamente diferencias significativas entre un método y otro utilizado en la resolución de problemas.

En consecuencia, de acuerdo, a las dos estadísticas utilizadas, muestran que las diferencias entre el grupo experimental es superior al grupo control.

La influencia que tendrá el taller de resolución de problemas, en la prueba integral, de cada unidad, se detalla en la tabla 4. Comparando los resultados de rendimiento académico entre grupo control y experimental.

Tabla 4 - Resultados comparativos de las prueba Integrales por cada unidad.

\begin{tabular}{|ll|c|c|c|c|}
\hline & Grupo & $\mathrm{N}$ & Media & Desviación típ. & \% Rendimiento \\
\hline \multirow{2}{*}{1 PI } & control & 48 & $\mathbf{3 , 6}$ & 1,17 & $43 \%$ \\
& experimental & 24 & $\mathbf{3 , 6}$ & 0,76 & $43 \%$ \\
\hline \multirow{2}{*}{2 PI } & control & 48 & $\mathbf{4 , 5}$ & 0,17 & $58 \%$ \\
& experimental & 24 & $\mathbf{4 , 9}$ & 0,27 & $65 \%$ \\
\hline \multirow{2}{*}{3 PI } & control & 48 & $\mathbf{4 , 1}$ & 0,73 & $52 \%$ \\
& experimental & 24 & $\mathbf{4 , 8}$ & 0,83 & $63 \%$ \\
\hline
\end{tabular}

La tabla 4, se puede representar gráficamente, de manera darle una mejor interpretación a los resultados.

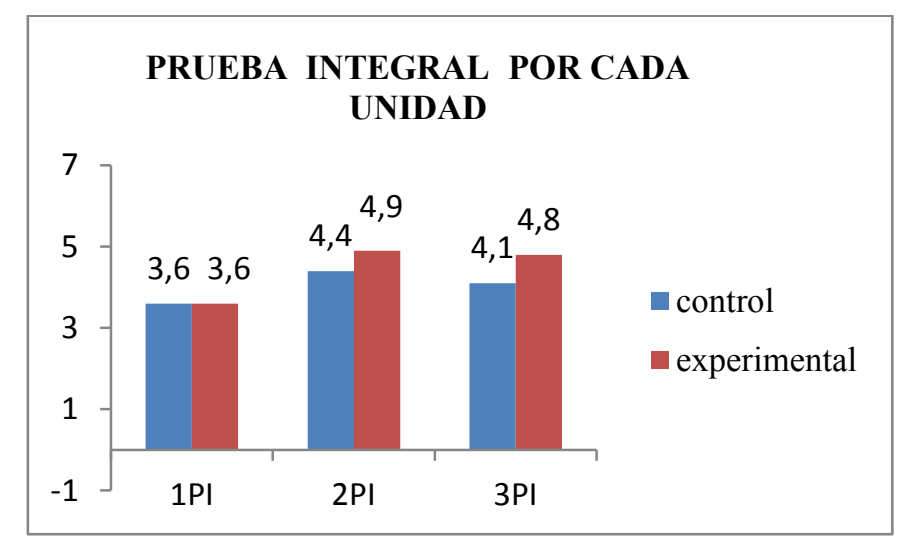

Gráfico 2 - Comparación de resultados de aprendizaje de prueba integral para cada unidad. 
Para asegura las diferencias en rendimiento y aprendizaje de cada unidad, se utiliza al igual que el caso anterior, la prueba paramétrica " $t$ ", mostrada en la tabla 5.

Tabla 5 - Prueba " $t$ " entre grupo control y experimental, para prueba integral de unidad temática.

\begin{tabular}{|c|c|c|c|}
\hline & "t" & $\mathrm{gl}$ & Diferencias media \\
\hline 1 Prueba Integral & $\mathbf{0 , 1 4}$ & 70 & $-0,04$ \\
\hline 2 Prueba Integral & $\mathbf{1 , 5 9}$ & 70 & $-0,50$ \\
\hline 3 Prueba Integral & $\mathbf{3 , 9 1}$ & 70 & $-0,74$ \\
\hline
\end{tabular}

Para 70 grados de libertad la prueba "t", tiene un valor $-1,67<\mathrm{t}<1,67$. Por lo tanto, queda claro que la propuesta, tiene diferencias que van aumentando en la medida que se avanza por unidad temática.

Como, las condiciones que deben aplicarse a la prueba "t" no se cumplen nítidamente, se hace confirma con la prueba no paramétrica "U" de Mann-Whitney. Los resultados se muestran en la siguiente tabla.

Tabla 6 - Estadísticos de contraste(a) prueba "U", compara resultado de la prueba integral entre grupo control y el experimental.

\begin{tabular}{|l|c|c|c|}
\hline & $\begin{array}{c}\text { Primera Prueba } \\
\text { Integral }\end{array}$ & $\begin{array}{c}\text { Segunda Prueba } \\
\text { Integral }\end{array}$ & $\begin{array}{c}\text { Tercera Prueba } \\
\text { Integral }\end{array}$ \\
\hline U de Mann-Whitney & 573,500 & 453,000 & 292,000 \\
W de Wilcoxon & 1798,500 & 1678,000 & 1517,000 \\
$\mathbf{Z}$ & $\mathbf{- 0 , 1 7}$ & $\mathbf{- 1 , 5 9}$ & $\mathbf{- 3 , 4 8}$ \\
Sig. asintót. (bilateral) & 0,865 & 0,113 & 0,000 \\
\hline
\end{tabular}

Como se trata de muestra grandes, mayores a 20. El valor de $\mathrm{Z}$ debe estar en el intervalo entre $-1,96<Z<1,96$. Como se observa en la primera prueba integral no aparecen diferencia, en la segunda prueba integral se observa diferencia, pero la prueba estadística no asegura que sea de la propuesta, en la tercera prueba integral si se nota diferencias importantes entre grupo control y experimental.

\section{Conclusiones}

En primer lugar, se puede concluir que la metodología indagatoria guiada con apoyo de las tecleras o clickers se obtiene mejores resultados de rendimiento que los obtenidos mediante el grupo control, en los talleres de resolución de problema en grupo de manera tradicional. En la primera unidad las evaluaciones de rendimiento de los talleres de resolución 


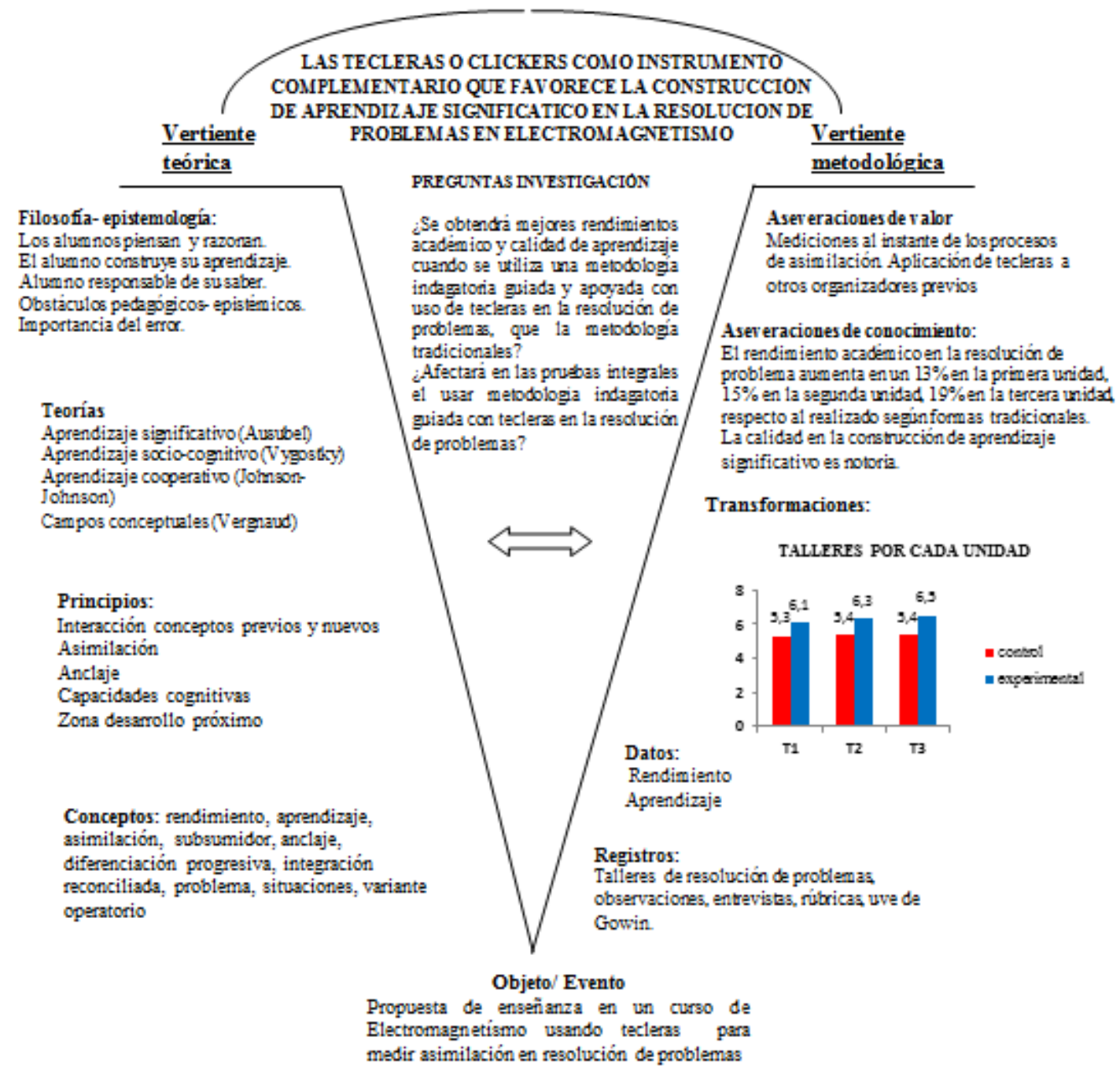

de problemas son un 13\% mejores para el grupo experimental sobre el grupo control, en la segunda unidad son del $15 \%$ y en la tercera unidad un $19 \%$ toda a favor del grupo experimental, como lo señala el gráficol. El taller de resolución de problemas con uso de tecleras y su incidencia en la construcción de aprendizaje significativo es relevante, porque permite que los estudiantes vayan midiendo permanentemente su aprendizaje, negociando significado con sus compañeros y con el profesor, asegurando un anclaje en forma adecuada. Todo ello, a través de su leguaje y sus respuestas que son detectadas y grabadas en las tecleras.

En segundo lugar, se puede concluir, que la metodología utilizada en la resolución de problemas está relacionada con un aumento del rendimiento y aprendizaje en las pruebas integrales. En la primera prueba integral no se observa diferencia en el rendimiento académico, en la segunda unidad temática la diferencia es del 7\% y en la tercera unidad $11 \%$, siempre a favor del grupo experimental, como lo muestra el gráfico 2. Al concluir sobre los aprendizajes logrado por cada una de las unidades se puede establecer de la información de los mapas conceptuales que los alumnos deben hacer en cada unidad y de los procedimientos 
utilizados en la resolución de cada unidad, un aumento paulatino a medida que se avanzan con los contenidos del programa de curso, hecho que ocurre en los talleres de resolución de problemas así como en las pruebas integrales.

Este aumento gradual se explica en el hecho de como los estudiantes se van apropiando con la metodología de enseñanza que se propone para la resolución de problema. Es claro, que las tecleras o clickers juegan un rol relevante en la promoción de aprendizaje significativo en electromagnetismo, en especial en la resolución de problemas.

\section{Agradecimientos}

Convenio UPA 1203, Convenio Desempeño de la Universidad de Playa Ancha.

\section{Referencias}

AUSUBEL, D. P. The acquisition and retention of knowledge: A cognitive view. Dordrecht: Kluwer Academic Publishers, 2000.

BENEGAS, J. Tutoriales para Física Introductoria: Una experiencia exitosa de Aprendizaje Activo de la Física. Latin-American Journal of Physics Education, v. 1, n. 1, 2007.

GUISASOLA, J.; ZUBIMENDI, J. L.; ALMUDI, J. M.; CEBERIO, M. Propuesta de Enseñanza en Cursos Introductorios de Física en la Universidad basada en investigación didáctica. Enseñanza de las Ciencias, v. 25, p. 91-106, 2007.

McDEMONTT, L. C.; SHAFFER, P. S. Tutoriales para Física Introductoria. Buenos Aires: Prentice Hall, 2001.

MOREIRA, M A. Investigación Básica en Educación en Ciencias: Una Visión Personal. Revista Chilena de Educación Científica, v. 3, n. 1, p. 10-17, 2004.

MOREIRA, M. A. Fundamentos Teóricos para la Investigación en Enseñanza de las Ciencias, In: PROGRAMA INTERNACIONAL DE DOCTORADO EN ENSEÑANZA DE LAS CIENCIAS (PIDEC), Universidad de Burgos, España, 1999. Actas...

MOREIRA, M. A. A teoria da aprendizagem significativa e sua implementação em sala de aula. Brasília: Editora da UnB, 2006.

MOREIRA, M. A. Teorias de aprendizagem. São Paulo: E. P. U., 2011. 242p 
QUINN, A. An exploratory study of opinions on clickers and class participation from students of human behaviour in the social environment. Journal of Human Behaviour in the Social Environment, v. 20, n. 6, p. 721-731, 2010.

SILVA, R. La enseñanza de la Física mediantes un aprendizaje significativo y cooperativo en Blended Learning. 2011. Tesis (Doctorado) - Universidad de Burgos, Espanha.

SILVA, R.; LÓPEZ, E. Metodología activa para la enseñanza de la Física con apoyo de elearning. Visiones Científicas, Valparaíso, v. 7, n. 1, 2004.

SILVA, R.; LÓPEZ, E. Modelo metodológico didáctico para la enseñanza de la Física Experimental ambientado en Blended Learning. Visiones Científicas, Valparaíso, v. 8, n. 1, 2009.

SORIANO, M. R.; BARBIRIC, D. A.; SPELTINI, C. Método de indagación guiada en cursos de Química General. Análisis de casos. In: JORNADAS DE ENSEÑANZA UNIVERSITARIA DE LA QUÍMICA, VII, 2006. Actas... p. 21-26. 